\title{
Nonlinear Stability of a Cylindrical Interface with Mass and Heat Transfer
}

\author{
Doo-Sung Lee \\ Department of Mathematics (Education), College of Education, Konkuk University, 93-1, \\ Mojin-Dong, Kwangjin-Gu, Seoul, Korea \\ Reprint requests to Prof. D.-S. L.; Fax: 001-82-454-3864; E-mail: dslee@kkucc.konkuk.ac.kr
}

Z. Naturforsch. 55a, 837-842 (2000); received November 29, 1999

\begin{abstract}
The nonlinear Rayleigh-Taylor stability of a cylindrical interface between vapor and the liquid phases of a fluid is studied when the phases are enclosed between two cylindrical surfaces coaxial with the interface, and when there is mass and heat transfer across the interface. The method of multiple time scale expansion is used for the investigation. A simple nondimensional parameter is found to characterize the stability of the system. Using this parameter, the region of stability is displayed graphically.
\end{abstract}

Key words: Rayleigh-Taylor Stability; Mass and Heat Transfer; Cylindrical Interface.

\section{Introduction}

The problem of stability of liquids when there is mass an heat transfer across the interface has been investigated in [1-7]. Hsieh [2] established a general formulation of the interfacial flow problem with mass and heat transfer and applied it to the Rayleigh-Taylor and KelvinHelmholtz instability problems in plane geometry. In the nuclear reactor cooling of fuel rods by liquid coolants, the geometry of the system in many cases is cylindrical. Indeed, there have been some investigations $[6,7]$ on the stability of a cylindrical interface between two fluids when there is a mass and heat transfer across the interface. The analysis of these studies was confined within the framework of the linear theory.

The effect of mass and heat transfer across the interface should be taken into account in the stability discussions, when the situation is like film boiling of fluids. However, with the linear analysis, the stability criteria remain the same as in the case with the neglect of heat and mass transfer across the interface. Hsieh found [1] that when the vapor region is hotter than the liquid region, as is usually so, the effect of mass and heat transfer tends to inhibit the growth of the instability. Thus, it is clear that such a uniform model based on the linear theory is inadequate to explain the mechanism involved, and the nonlinear theory is needed to reveal the effect of heat and mass transfer on the stability of the system. This problem is of fundamental importance in a number of applications such as design of many types of contacting equipment, e.g., boilers, condensers and reactors in industrial and environmental processes.
The purpose of this paper is to investigate the nonlinear stability of a cylindrical interface between vapor and liquid phases of a fluid when there is a mass and heat transfer across the interface. The basic equations with the accompanying boundary conditions are given in Sect. 2 . The first order the linear dispersion relation are obtained in Sect. 3. In Sect. 4, we have derived solutions near the linear critical wavenumber. In Sect. 5, some numerical examples are presented in graphical form.

\section{Formulation of the Problem and Basic Equations}

We shall use a cylindrical system of coordinates $(r, \theta$, $z$ ) so that in the equilibrium state the $z$-axis is the axis of symmetry of the system. The central solid core has a radius $a$. In the equilibrium state the fluid phase " 1 ", of density $\rho^{(1)}$, occupies the region $a<r<R$, and, the fluid phase " 2 ", of density $\rho^{(2)}$, occupies the region $R<r<b$. The temperatures at $r=a, R$, and $b$ are taken as $T_{1}, T_{0}$, and $T_{2}$, respectively. The bounding surfaces $r=a$, and $r=b$ are taken as rigid. The interface, after a disturbance is given by

$$
F(r, z, t)=r-R-\eta=0,
$$

where $\eta$ is the perturbation in radius of the interface from its equilibrium value $R$, and for which the outward normal vector is written as

$$
\boldsymbol{n}=\frac{\nabla F}{|\nabla F|}=-\left\{1+\left(\frac{\partial \eta}{\partial z}\right)^{2}\right\}^{-1 / 2}\left(\boldsymbol{e}_{r}-\frac{\partial \eta}{\partial z} \boldsymbol{e}_{z}\right) .
$$


We assume that fluid velocity is irrotational so that the velocity potentials are $\phi^{(1)}$ and $\phi^{(2)}$ for the fluid phases 1 and 2. In each fluid phase

$$
\nabla^{2} \phi^{(j)}=0, \quad(j=1,2) .
$$

The solutions for $\phi^{(j)}(j=1,2)$ have to satisfy the boundary conditions. The relevant boundary conditions for our configuration are

(i) On the rigid boundaries $r=a$ and $r=b$ :

The normal field velocities vanish on both the central solid core and the outer bounding surface:

$$
\begin{aligned}
& \frac{\partial \phi^{(1)}}{\partial r}=0 \quad \text { on } r=a, \\
& \frac{\partial \phi^{(2)}}{\partial r}=0 \quad \text { on } r=b .
\end{aligned}
$$

(ii) On the interface $r=R+\eta(z, t)$ :

I. The conservation of mass across the interface requires

$$
\begin{aligned}
& \llbracket \rho\left(\frac{\partial F}{\partial t}+\nabla \phi \cdot \nabla F\right) \rrbracket=0, \\
& \text { or } \llbracket \rho\left(\frac{\partial \phi}{\partial r}-\frac{\partial \eta}{\partial t}-\frac{\partial \eta}{\partial z} \frac{\partial \phi}{\partial z}\right) \rrbracket=0,
\end{aligned}
$$

where $\llbracket \rrbracket$ represents the difference in a quantity as we cross the interface, i.e., $\llbracket h \rrbracket=h^{(2)}-h^{(1)}$, where the superscripts refer to the upper and lower fluids, respectively.

II. The interfacial condition for energy is

$$
L \rho^{(1)}\left(\frac{\partial F}{\partial t}+\nabla \phi^{(1)} \cdot \nabla F\right)=S(\eta),
$$

where $L$ is the latent heat released when the fluid is transformed from phase 1 to phase 2. Physically, the left-hand side of (7) represents the latent heat released during the phase transformation, while $S(\eta)$ on the right-hand side of (7) represents the net heat flux, so that the energy will be conserved.

In the equilibrium state, the heat fluxes in the direction of increasing $r$ in the fluid phases 1 and 2 are $-K_{1}\left(T_{1}-T_{0}\right) / R \log (a / R)$ and $-K_{2}\left(T_{0}-T_{2}\right) / R \log (R / b)$, where $K_{1}$ and $K_{2}$ are the heat conductivities of the two fluids. As in [2], we write

$$
\begin{aligned}
S(\eta) & =\frac{K_{2}\left(T_{0}-T_{2}\right)}{(R+\eta)(\log b-\log (R+\eta))} \\
& -\frac{K_{1}\left(T_{1}-T_{0}\right)}{(R+\eta)(\log (R+\eta)-\log a)},
\end{aligned}
$$

and we expand it about $r=R$ by Taylor's expansion, such that

$$
S(\eta)=S(0)+\eta S^{\prime}(0)+\frac{1}{2} \eta^{2} S^{\prime \prime}(0)+\ldots,
$$

and we take $S(0)=0$, so that

$$
\frac{K_{2}\left(T_{0}-T_{2}\right)}{R \log (b / R)}=\frac{K_{1}\left(T_{1}-T_{0}\right)}{R \log (R / a)}=G(\text { say }),
$$

indicating that in the equilibrium state the heat fluxes are equal across the interfaces in the two fluids.

From (1), (7), and (9), we have

$$
\begin{array}{r}
\rho^{(1)}\left(\frac{\partial \phi^{(1)}}{\partial r}-\frac{\partial \eta}{\partial t}-\frac{\partial \eta}{\partial z} \frac{\partial \phi^{(1)}}{\partial z}\right) \\
\quad=\alpha\left(\eta+\alpha_{2} \eta^{2}+\alpha_{3} \eta^{3}\right),
\end{array}
$$

where

$$
\begin{aligned}
& \alpha=\frac{G \log (b / a)}{L R \log (b / R) \log (R / a)}, \\
& \alpha_{2}=\frac{1}{R}\left(-\frac{3}{2}+\frac{1}{\log (b / R)}-\frac{1}{\log (R / a)}\right) .
\end{aligned}
$$

III. The conservation of momentum balance, by taking into account the mass transfer across the interface, is

$$
\begin{aligned}
\rho^{(1)}\left(\nabla \phi^{(1)} \cdot \nabla F\right)\left(\frac{\partial F}{\partial t}+\nabla \phi^{(1)} \cdot \nabla F\right) \\
=\rho^{(2)}\left(\nabla \phi^{(2)} \cdot \nabla F\right)\left(\frac{\partial F}{\partial t}+\nabla \phi^{(2)} \cdot \nabla F\right) \\
\quad+\left(p_{2}-p_{1}+\sigma \nabla \cdot \boldsymbol{n}\right)|\nabla F|^{2},
\end{aligned}
$$

where $p$ is the pressure and $\sigma$ the surface tension, respectively.

By eliminating the pressure by Bernoulli's equation we can rewrite the above condition (12) as

$$
\begin{gathered}
\llbracket \rho\left\{\frac{\partial \phi}{\partial t}+\frac{1}{2}\left(\frac{\partial \phi}{\partial r}\right)^{2}+\frac{1}{2}\left(\frac{\partial \phi}{\partial z}\right)^{2}-\left\{1+\left(\frac{\partial \eta}{\partial z}\right)^{2}\right\}^{-1}\right. \\
\left.\left.\quad \times\left(\frac{\partial \phi}{\partial z} \frac{\partial \eta}{\partial z}-\frac{\partial \phi}{\partial r}\right)\left(\frac{\partial \eta}{\partial t}+\frac{\partial \phi}{\partial z} \frac{\partial \eta}{\partial z}-\frac{\partial \phi}{\partial r}\right)\right\}\right] \\
=-\sigma \frac{\partial^{2} \eta}{\partial z^{2}}\left\{1+\left(\frac{\partial \eta}{\partial z}\right)^{2}\right\}^{-3 / 2} \\
+\sigma(R+\eta)^{-1}\left\{1+\left(\frac{\partial \eta}{\partial z}\right)^{2}\right\}^{-1 / 2}
\end{gathered}
$$

To investigate the nonlinear effects on the stability of the system, we employ the method of multiple scales (Lee 
[8-10]). Introducing $\varepsilon$ as a small parameter, we assume the following expansion of the variables:

$$
\begin{aligned}
& \eta=\sum_{n=1}^{3} \varepsilon^{n} \eta_{n}\left(z, T_{0}, T_{1}, T_{2}\right)+O\left(\varepsilon^{4}\right), \\
& \phi^{(j)}=\sum_{n=1}^{3} \varepsilon^{n} \phi_{n}^{(j)}\left(r, z, T_{0}, T_{1}, T_{2}\right)+O\left(\varepsilon^{4}\right), \\
& (j=1,2) .
\end{aligned}
$$

The quantities appearing in the field equations (3) and the boundary conditions (6), (11), and (13) can now be expressed in Maclaurin series expansion around $r=R$. Then, we use (14), and (15) and equate the coefficients of equal power series in $\varepsilon$ to obtain the linear and the successive nonlinear partial differential equations of a various orders (see Appendix).

\section{Linear Theory}

The linear wave solutions of (3), subject to the boundary conditions, yield

$$
\begin{aligned}
& \eta_{1}=A\left(T_{1}, T_{2}\right) e^{i \theta}+\bar{A}\left(T_{1}, T_{2}\right) e^{-i \theta}, \\
& \phi_{1}^{(1)}=\frac{1}{k}\left(\frac{\alpha}{\rho^{(1)}}-i \omega\right) A\left(T_{1}, T_{2}\right) E_{1}(k r) e^{i \theta}+c . c ., \\
& \phi_{1}^{(2)}=\frac{1}{k}\left(\frac{\alpha}{\rho^{(2)}}-i \omega\right) A\left(T_{1}, T_{2}\right) E_{2}(k r) e^{i \theta}+c . c .,
\end{aligned}
$$

where

$$
\begin{aligned}
& E_{1}(k r)=\frac{I_{0}(k r) K_{1}(k a)+I_{1}(k a) K_{0}(k r)}{I_{1}(k R) K_{1}(k a)-I_{1}(k a) K_{1}(k R)}, \\
& E_{2}(k r)=\frac{I_{0}(k r) K_{1}(k b)+I_{1}(k b) K_{0}(k r)}{I_{1}(k R) K_{1}(k b)-I_{1}(k b) K_{1}(k R)}, \\
& \theta=k z-\omega T_{0}
\end{aligned}
$$

with $I_{m}$ and $K_{m}(m=0,1)$ the modified Bessel functions of the first and second kinds, respectively.

Substituting (16)-(18) into (A.3), we obtain the dispersion relation

$$
a_{0} \omega_{0}^{2}+a_{1} \omega_{0}+a_{2}=0
$$

where

$$
\begin{aligned}
& a_{0}=\rho^{(1)} E_{1}(k R)-\rho^{(2)} E_{2}(k R), \\
& a_{1}=\alpha\left\{E_{1}(k R)-E_{2}(k R)\right\}, \\
& a_{2}=\frac{\sigma k}{R^{2}}\left(R^{2} k^{2}-1\right), \omega_{0}=-i \omega .
\end{aligned}
$$

From the properties of Bessel functions, and since $\alpha$ is always positive, we notice that $a_{0}>0$, and $a_{1}>0$. Applying the Routh-Hurwitz criteria to (22), the condition for stability is $a_{2}>0$, from which we obtain $k>\frac{1}{R}$. Thus the system is stable if $k>k_{c}$, where

$$
k_{c}=\frac{1}{R} .
$$

The corresponding critical frequency, $\omega_{c}$ is zero for this case.

\section{Solutions near the Linear Critical Wavenumber}

To solve equations in the neighborhood of the linear critical wave number $k_{c}$, we assume that the critical wavenumber, because of nonlinear effect, will shift to

$$
k=k_{c}+\varepsilon^{2} \mu .
$$

The first-order solution will reproduce the linear results for the critical case, and they are obtained from (16)-(18) by setting $\omega$ equal to zero.

With the use of the first order solutions for the critical wavenumber, we obtain the equations for the second order problem

$$
\nabla^{2} \phi_{2}^{(j)}=0, \quad(j=1,2) .
$$

and the boundary conditions at $r=R$ :

$$
\begin{aligned}
\rho^{(1)} \frac{\partial \phi_{2}^{(1)}}{\partial r} & -\alpha \eta_{2}-\frac{\partial \eta_{2}}{\partial T_{0}} \\
& =\rho^{(1)} \frac{\partial A}{\partial T_{1}} e^{i k z} \\
& +\alpha\left\{\frac{1}{R}-2 k E_{1}(k R)+\alpha_{2}\right\} A^{2} e^{2 i k z} \\
& + \text { c.c. }+2 \alpha\left(\frac{1}{R}+\alpha_{2}\right)|A|^{2}
\end{aligned}
$$

$$
\begin{aligned}
\rho^{(2)} \frac{\partial \phi_{2}^{(2)}}{\partial r}-\alpha \eta_{2}-\frac{\partial \eta_{2}}{\partial T_{0}} \\
=\rho^{(2)} \frac{\partial A}{\partial T_{1}} e^{i k z} \\
\quad+\alpha\left\{\frac{1}{R}-2 k E_{2}(k R)+\alpha_{2}\right\} A^{2} e^{2 i k z} \\
\quad+\text { c.c. }+2 \alpha\left(\frac{1}{R}+\alpha_{2}\right)|A|^{2},
\end{aligned}
$$




$$
\begin{aligned}
\rho^{(1)} & \frac{\partial \phi_{2}^{(1)}}{\partial T_{0}}-\rho^{(2)} \frac{\partial \phi_{2}^{(2)}}{\partial T_{0}}-\sigma\left[\frac{\partial^{2} \eta_{2}}{\partial z^{2}}+\frac{\eta_{2}}{R^{2}}\right] \\
= & \left\{\rho^{(2)} E_{2}(k R)-\rho^{(1)} E_{1}(k R)\right\} \frac{\partial A}{\partial T_{1}} e^{i k z} \\
& +\alpha^{2} \frac{1}{2}\left\{\frac{1+E_{1}^{2}(k R)}{\rho^{(1)}}-\frac{1+E_{2}^{2}(k R)}{\rho^{(2)}}\right\} A^{2} e^{2 i k z} \\
& -\frac{\sigma}{2 R^{3}}\left(R^{2} k^{2}+2\right) A^{2} e^{2 i k z} \\
& + \text { c.c. }+\alpha^{2}\left\{\frac{1-E_{1}^{2}(k R)}{\rho^{(1)}}-\frac{1-E_{2}^{2}(k R)}{\rho^{(2)}}\right\}|A|^{2} \\
& +\frac{\sigma}{R^{3}}\left(R^{2} k^{2}-2\right)|A|^{2},
\end{aligned}
$$

The non secularity conditions for the existence of the uniformly valid solution are

$$
\frac{\partial A}{\partial T_{1}}=0
$$

and its complex conjugate relation.

Equations (26) to (28) furnish the second order solutions

$$
\eta_{2}=-2\left(\frac{1}{R}+\alpha_{2}\right)|A|^{2}+A_{2} e^{2 i k z}+\bar{A}_{2} e^{-2 i k z},
$$

$$
\begin{aligned}
\phi_{2}^{(1)}= & b^{(1)}\left(T_{0}, T_{1}, T_{2}\right) \\
& +\left(B_{2} e^{2 i k z}+\bar{B}_{2} e^{-2 i k z}\right) E_{1}(2 k r), \\
\phi_{2}^{(2)}= & b^{(2)}\left(T_{0}, T_{1}, T_{2}\right) \\
& +\left(C_{2} e^{2 i k z}+\bar{C}_{2} e^{-2 i k z}\right) E_{2}(2 k r)
\end{aligned}
$$

where

$$
E_{1}(2 k r)=\frac{I_{0}(2 k r) K_{1}(2 k a)+I_{1}(2 k a) K_{0}(2 k r)}{I_{1}(2 k R) K_{1}(2 k a)-I_{1}(2 k a) K_{1}(2 k R)},
$$

$$
E_{2}(2 k r)=\frac{I_{0}(2 k r) K_{1}(2 k b)+I_{1}(2 k b) K_{0}(2 k r)}{I_{1}(2 k R) K_{1}(2 k b)-I_{1}(2 k b) K_{1}(2 k R)},
$$

$$
\begin{aligned}
& A_{2}=N A^{2}, \\
& B_{2}=\frac{\alpha A^{2}}{2 \rho^{(1)}}\left[\frac{N}{k}+\frac{1}{k R}-2 E_{1}(k R)+\frac{\alpha_{2}}{k}\right], \\
& C_{2}=\frac{\alpha A^{2}}{2 \rho^{(2)}}\left[\frac{N}{k}+\frac{1}{k R}-2 E_{2}(k R)+\frac{\alpha_{2}}{k}\right],
\end{aligned}
$$

$$
\begin{aligned}
N= & \frac{R^{2} \alpha^{2}}{\left(8 k^{2} R^{2}-2\right) \sigma} \\
& \cdot\left\{\frac{1+E_{1}^{2}(k R)}{\rho^{(1)}}-\frac{1+E_{2}^{2}(k R)}{\rho^{(2)}}\right\} \\
& -\frac{k^{2} R^{2}+2}{\left(8 k^{2} R^{2}-2\right) R}, \\
\rho^{(1)} & \frac{\partial b^{(1)}}{\partial T_{0}}-\rho^{(2)} \frac{\partial b^{(2)}}{\partial T_{0}} \\
= & {\left[-\alpha^{2}\left\{\frac{-1+E_{1}^{2}(k R)}{\rho^{(1)}}-\frac{-1+E_{2}^{2}(k R)}{\rho^{(2)}}\right\}\right.} \\
& \left.+\frac{\sigma}{R^{3}}\left(k^{2} R^{2}-2\right)\right]|A|^{2}-2 \alpha\left(\frac{1}{R}+\alpha_{2}\right)|A|^{2},
\end{aligned}
$$

We substitute the first- and second-order solutions into the third order equation. In order to avoid nonuniformity of the expansion, we again impose the condition that secular terms vanish. Then, from (A.9), we find

$$
\begin{aligned}
\left\{E_{1}(k R)\right. & \left.-E_{2}(k R)\right\} \frac{\alpha}{k} \frac{\partial A}{\partial T_{2}} \\
& +2 \sigma k_{c} \mu A+v|A|^{2} A=0,
\end{aligned}
$$

where

$$
\begin{aligned}
v= & \alpha^{2}\left\{\left(N+\frac{1}{R}+\alpha_{2}\right)\right. \\
& \cdot\left[\frac{E_{1}(k R) E_{1}(2 k R)-1}{\rho^{(1)}}-\frac{E_{2}(k R) E_{2}(2 k R)-1}{\rho^{(2)}}\right] \\
& -2 k\left[\frac{E_{1}(k R)}{\rho^{(1)}}\left\{E_{1}(k R) E_{1}(2 k R)-1\right\}-\frac{E_{2}(k R)}{\rho^{(2)}}\right. \\
& \left.\left.\cdot\left\{E_{2}(k R) E_{2}(2 k R)-1\right\}\right]+\frac{3}{R}\left(\frac{1}{\rho^{(1)}}-\frac{1}{\rho^{(2)}}\right)\right\}-\frac{\sigma}{R^{4}} \\
& \cdot\left\{-R 2 N\left(1-k^{2} R^{2}\right)+4 R \alpha_{2}\right. \\
& \left.+7-\frac{1}{2} k^{2} R^{2}\left(1-3 k^{2} R^{2}\right)\right\} .
\end{aligned}
$$

\section{Discussion}

There is no loss of generality to treat $A$ as real in (40) since the phase associated with $A$ remains constant. Thus we may rewrite (40) as

$$
\frac{\mathrm{d} A}{\mathrm{~d} T_{2}}+\left(a_{1}+a_{2} A^{2}\right) A=0
$$


where

$$
a_{1}=\frac{2 \sigma \mu k_{c} k}{\alpha\left\{E_{1}(k R)-E_{2}(k R)\right\}}
$$

and

$$
a_{2}=\frac{v k}{\alpha\left\{E_{1}(k R)-E_{2}(k R)\right\}} .
$$

Denoting $A(0)$ by $A_{0}$, we obtain

$$
\begin{aligned}
A^{2}\left(T_{2}\right)=a_{1} A_{0}^{2} \exp \left(-2 a_{1} t\right) \\
\cdot\left[a_{1}+a_{2} A_{0}^{2}-a_{2} A_{0}^{2} \exp \left(-2 a_{1} t\right)\right]^{-1} .
\end{aligned}
$$

With a finite value of $A_{0}$, let us consider the values of $a_{1}$ and $a_{2}$ for which the denominator in (45) is not equal to zero. In this case $A$ is asymptotically bounded.

From (45), we can see that if $a_{2}>0$, the system is always stable. The stability condition $a_{2}>0$ is equivalent to

$$
v>0 \text {. }
$$

Since the vapor density $\rho^{(1)}$ is usually much smaller than the liquid density $\rho^{(2)}$, when $\rho^{(1)} \ll \rho^{(2)}$, $v$ is approximately

$$
\begin{aligned}
v= & \frac{\alpha^{2}}{\rho^{(1)}}\left\{\left\{E_{1}(k R) E_{1}(2 k R)-1\right\}\right. \\
& \left.\cdot\left\{N+\frac{1}{2}+\alpha_{2}-2 k E_{1}(k R)\right\}+\frac{3}{R}\right\} \\
& +\frac{\sigma}{R^{4}}\left\{R 2 N\left(1-k^{2} R^{2}\right)-4 R \alpha_{2}-7\right. \\
& \left.-\frac{1}{2} k^{2} R^{2}\left(1-3 k^{2} R^{2}\right)\right\},
\end{aligned}
$$

where

$$
N=\frac{1}{R}\left[\frac{R^{3} \alpha^{2}}{\rho^{(1)} \sigma} \frac{1+E_{1}^{2}(k R)}{\left(8 k^{2} R^{2}-2\right)}-\frac{k^{2} R^{2}+2}{\left(8 k^{2} R^{2}-2\right)}\right] .
$$

From the criteria (46) and (47) it is seen that a relevant nondimensional parameter is

$$
\delta=\frac{R^{3} \alpha^{2}}{\varrho^{(1)} \sigma},
$$

and the condition (46) can be written in the form

$$
\mathscr{A} \delta^{2}+\mathscr{B} \delta+\mathscr{C}>0 \text {, }
$$

from which we can obtain the value $\delta$ for which the system is stable. In Fig. 1, we show the variation of $\delta$ with respect to the thickness of the vapor $h_{1}$. Here we have chosen $a=1$ and $b \gg a$. The region above the curve is the stable region, while below the curve is the region

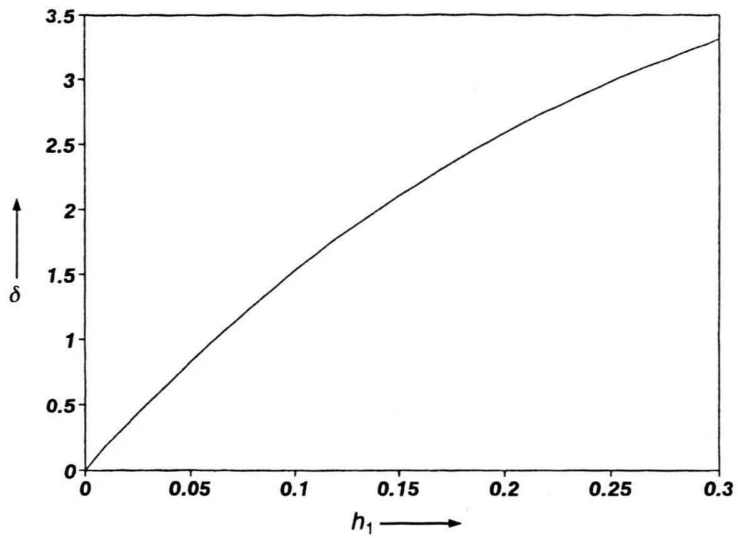

Fig. 1. The variation of $\delta$ with respect to the thickness $h_{1}$.

of instability. From this figure we see that as $h_{1}$ increase, $\delta$ increases also. This means, since $\alpha$ is proportional to the heat flux of the system: with the same heat flux, the thinner the vapor the easier the system can be stabilized.

When the system is linearly unstable, i.e., when $\mu<0$, the asymptotic value of $A$ for larger times will be given by

$$
|A|^{2}=-2 \sigma k_{c} \mu / v \text {. }
$$

From practical considerations, we would expect that as the amplitude exceeds the thickness of the fluid layers, there is a tendency for bubbles to form and detach from the interface, as to cause the rupture of the fluid layers.

The criterion against the rupture is given by

$$
h_{1}^{2}>-2 \sigma k_{c} \mu / v \text {. }
$$

In the case $a_{2}<0$, the stability can also be established if $a_{1}>0$ and the initial amplitude is small enough, i.e., $A_{0}<-a_{1} / a_{2}$.

\section{Appendix}

The interfacial conditions are given on $r=R$ as

Order $O(\varepsilon)$

$$
\begin{aligned}
& \left.\llbracket \rho\left(\frac{\partial \phi_{1}}{\partial r}-\frac{\partial \eta_{1}}{\partial T_{0}}\right)\right]=0, \\
& \rho^{(1)}\left(\frac{\partial \phi_{1}^{(1)}}{\partial r}-\frac{\partial \eta_{1}}{\partial T_{0}}\right)=\alpha \eta_{1}, \\
& \llbracket \rho \frac{\partial \phi_{1}}{\partial T_{0}} \rrbracket=-\sigma\left(\frac{\partial^{2} \eta_{1}}{\partial z^{2}}+\frac{\eta_{1}}{R^{2}}\right) .
\end{aligned}
$$


Order $O\left(\varepsilon^{2}\right)$

$$
\begin{aligned}
& \left.\llbracket \rho\left(\frac{\partial \phi_{2}}{\partial r}+\frac{\partial^{2} \phi_{1}}{\partial r^{2}} \eta_{1}-\frac{\partial \eta_{2}}{\partial T_{0}}-\frac{\partial \eta_{1}}{\partial T_{1}}-\frac{\partial \eta_{1}}{\partial z} \frac{\partial \phi_{1}}{\partial z}\right)\right]=0, \\
& \rho^{(1)}\left(\frac{\partial \phi_{2}^{(1)}}{\partial r}+\frac{\partial^{2} \phi_{1}^{(1)}}{\partial r^{2}} \eta_{1}-\frac{\partial \eta_{2}}{\partial T_{0}}-\frac{\partial \eta_{1}}{\partial T_{1}}-\frac{\partial \eta_{1}}{\partial z} \frac{\partial \phi_{1}^{(1)}}{\partial z}\right)=\alpha\left(\eta_{2}+\alpha_{2} \eta_{1}^{2}\right), \\
& \left.\llbracket \rho\left\{\frac{\partial \phi_{2}}{\partial T_{0}}+\frac{\partial \phi_{1}}{\partial T_{1}}+\frac{\partial^{2} \phi_{1}}{\partial T_{0} \partial r} \eta_{1}+\frac{1}{2}\left[\left(\frac{\partial \phi_{1}}{\partial r}\right)^{2}+\left(\frac{\partial \phi_{1}}{\partial z}\right)^{2}\right]+\frac{\partial \phi}{\partial r}\left(\frac{\partial \eta_{1}}{\partial T_{0}}-\frac{\partial \phi_{1}}{\partial r}\right)\right\}\right] \\
& =-\sigma\left\{\frac{\partial^{2} \eta_{2}}{\partial z^{2}}+\frac{1}{2 R}\left(\frac{\partial \eta_{1}}{\partial z}\right)^{2}+\frac{\eta_{2}}{R^{2}}-\frac{\eta_{1}^{2}}{R^{3}}\right\} .
\end{aligned}
$$

Order $O\left(\varepsilon^{3}\right)$

$$
\begin{gathered}
\llbracket \rho\left\{\frac{\partial \phi_{3}}{\partial r}+\frac{\partial^{2} \phi_{2}}{\partial r^{2}} \eta_{1}+\frac{\partial^{2} \phi_{1}}{\partial r^{2}} \eta_{2}+\frac{1}{2} \frac{\partial^{3} \phi_{1}}{\partial r^{3}} \eta_{1}^{2}-\frac{\partial \eta_{3}}{\partial T_{0}}-\frac{\partial \eta_{2}}{\partial T_{1}}-\frac{\partial \eta_{1}}{\partial T_{2}}\right. \\
\left.-\frac{\partial \eta_{1}}{\partial z}\left(\frac{\partial \phi_{2}}{\partial z}+\frac{\partial^{2} \phi_{1}}{\partial z \partial r} \eta_{1}\right)-\frac{\partial \eta_{2}}{\partial z} \frac{\partial \phi_{1}}{\partial z}\right\} \rrbracket=0,
\end{gathered}
$$

$$
\begin{aligned}
\rho^{(1)}\{ & \frac{\partial \phi_{3}^{(1)}}{\partial r}+\frac{\partial^{2} \phi_{2}^{(1)}}{\partial r^{2}} \eta_{1}+\frac{\partial^{2} \phi_{1}^{(1)}}{\partial r^{2}} \eta_{2}+\frac{1}{2} \frac{\partial^{3} \phi_{1}^{(1)}}{\partial r^{3}} \eta_{1}^{2}-\frac{\partial \eta_{3}}{\partial T_{0}}-\frac{\partial \eta_{2}}{\partial T_{1}}-\frac{\partial \eta_{1}}{\partial T_{2}} \\
& \left.-\frac{\partial \eta_{1}}{\partial z}\left(\frac{\partial \phi_{2}^{(1)}}{\partial z}+\frac{\partial^{2} \phi_{1}^{(1)}}{\partial z \partial r} \eta_{1}\right)-\frac{\partial \eta_{2}}{\partial z} \frac{\partial \phi_{1}^{(1)}}{\partial z}\right\}=\alpha\left(\eta_{3}+2 \alpha_{2} \eta_{1} \eta_{2}+\alpha_{3} \eta_{1}^{3}\right),
\end{aligned}
$$

$$
\begin{aligned}
\llbracket \rho & \left\{\frac{\partial \phi_{3}}{\partial T_{0}}+\frac{\partial \phi_{2}}{\partial T_{1}}+\frac{\partial \phi_{1}}{\partial T_{2}}+\frac{\partial^{2} \phi_{1}}{\partial T_{0} \partial r} \eta_{2}+\left(\frac{\partial^{2} \phi_{1}}{\partial T_{1} \partial r}+\frac{\partial^{2} \phi_{2}}{\partial T_{0} \partial r}\right) \eta_{1}\right. \\
& +\frac{1}{2} \frac{\partial^{3} \phi_{1}}{\partial T_{0} \partial r^{2}} \eta_{1}^{2}+\frac{\partial \phi_{1}}{\partial r}\left(\frac{\partial \phi_{2}}{\partial r}+\frac{\partial^{2} \phi_{1}}{\partial r^{2}} \eta_{1}\right)+\frac{\partial \phi_{1}}{\partial z}\left(\frac{\partial \phi_{2}}{\partial z}+\frac{\partial^{2} \phi_{1}}{\partial r \partial z} \eta_{1}\right) \\
& -\left(\frac{\partial \phi_{1}}{\partial r}-\frac{\partial \eta_{1}}{\partial T_{0}}\right)\left(\frac{\partial \phi_{2}}{\partial r}-\frac{\partial \phi_{1}}{\partial z} \frac{\partial \eta_{1}}{\partial z}\right)+\frac{\partial \phi_{1}}{\partial r}\left(\frac{\partial \eta_{2}}{\partial T_{0}}+\frac{\partial \eta_{1}}{\partial T_{1}}-\frac{\partial \phi_{2}}{\partial r}+\frac{\partial \phi_{1}}{\partial z} \frac{\partial \eta_{1}}{\partial z}\right) \\
& \left.\left.+\eta_{1} \frac{\partial^{2} \phi_{1}}{\partial r^{2}}\left(\frac{\partial \eta_{1}}{\partial T_{0}}-\frac{\partial \phi_{1}}{\partial r}\right)+\eta_{1} \frac{\partial \phi_{1}}{\partial r}\left(\frac{\partial^{2} \eta_{1}}{\partial T_{o} \partial r}-\frac{\partial^{2} \phi_{1}}{\partial r^{2}}\right)\right\}\right] \\
& =-\sigma\left\{\frac{\partial^{2} \eta_{3}}{\partial z^{2}}+\left(k_{c}^{2}+2 k_{c} \mu\right) \frac{\partial^{2} \eta_{1}}{\partial z^{2}}-\frac{3}{2} \frac{\partial^{2} \eta_{1}}{\partial z^{2}}\left(\frac{\partial \eta_{1}}{\partial z}\right)^{2}\right. \\
& \left.-\frac{1}{2} \frac{\eta_{1}}{R^{2}}\left(\frac{\partial \eta_{1}}{\partial z}\right)^{2}+\frac{1}{R} \frac{\partial \eta_{1}}{\partial z} \frac{\partial \eta_{2}}{\partial z}+\frac{\eta_{3}}{R}-\frac{2 \eta_{1} \eta_{2}}{R^{3}}+\frac{\eta_{1}^{3}}{R^{4}}\right\} .
\end{aligned}
$$

[1] D. Y. Hsieh, Trans ASME, 94D, 156 (1972).

[2] D. Y. Hsieh, Phys. of Fluids, 21, 745 (1978).

[3] D. Y. Hsieh, Phys. of Fluids, 22, 1435 (1979).

[4] Gurpreet K. Gill, R. K. Chhabra, and S. K. Trehan, Z Naturforsch. 50a, 805 (1995).

[5] A. R. F. Elhefnawy, Z. Angew. Math. Mech. 77, 13 (1997).
[6] A. R. Nayak and B. B. Chakraborty, Phys. of Fluids, 27, 1937 (1984).

[7] A. R. F. Elhefnawy, Int. J. Engng. Sci. 32, 805 (1994)

[8] D.-S. Lee, Z. Naturforsch. 54a, 335 (1999).

[9] D.-S. Lee, J. Phys. II 7, 1045 (1997).

[10] D.-S. Lee, Z. Angew. Math. Mech. 79, 627 (1999). 